\title{
Advances in Hepatitis B Research: From Virology to Clinical Management (A Special Issue)
}

\section{Xuanyong Lu${ }^{1}$, Ke-Qin Hu${ }^{2}$}

1 Chief Scientist of Hepatitis B Foundation of America.

2 Director of Hepatology Services, University of California, Irvine.

Corresponding address: Xuanyong Lu, Drexel Institute for Biotechnology and Virology Research, Department of Microbiology and Immunology, School of Medicine, Drexel University. 700 East Butler Ave., Doylestown, PA, 18901 USA. Telephone: 215-4894906. Fax: 215-489-4920. E-mail: Xuanyong.lu@drexel.edu.

Received: 2004.12.01; Accepted: 2005.01.01; Published: 2005.01.05

\section{Editorial}

Hepatitis B virus (HBV) infection is one of the major human health problems worldwide. It is estimated that chronic HBV infection affects more than 350 million people globally, spreading in every continent in Asia, America, Europe, and Africa. Despite the huge number of HBV infected cases, it has not received enough attention as it should be in term of research funding and media coverage. In the past few years, significant advances have been made in molecular virology, pathogenesis and treatment of HBV infection. However, there still exist many challenges in HBV research, clinical management, and exploration of safe and effective therapies for chronic hepatitis B.

This special issue intends to provide a broad view and the update advances in HBV research from basic science to clinic management of HBV infection. For basic scientific research, two articles were selected focusing on topics involved in $\mathrm{HBeAg}$ mutant and its clinical implication, and the association of altered apoptosis with HBV infection and development of HCC. For clinical advances, six articles were selected focusing on molecular diagnosis, approaches for management of chronic HBV infection, immunomodulating therapy, liver transplantation, HBV epidemiology and prevention, and natural history. Since chronic HBV infection has different clinical presentation which needs a different approach to management, three of the six articles address the general principle of HBV management, immunotherapy, and liver transplant in HBV infected patients.

Topics in this issue touch important progresses of current HBV study. Authors have provided their expertise for the prospective research in the future, and we hope this will inspire the efforts on understanding, treating, and eventually eradicating the existence of HBV infection in Human.

\section{Conflict of interest}

The authors have declared that no conflict of interest exists. 